sei es deshalb, nach zwölf Monaten eine individuelle Risikostratifizierung vorzunehmen und gegebenenfalls die duale Plättchenhemmung ohne Pause fortzuführen, wobei die 60-mg-Dosis zweimal täglich durchaus erwogen werden sollte.

\section{Ist die ESC-Leitlinie überholt?}

In der jüngst aktualisierten ESC-Leitlinie wird eine länger als zwölf Monate dauernde duale Plättchenhemmung nur mit dem Evidenzgrad IIb empfohlen. Diese sehr zurückhaltende Einstellung sei, so Hamm, der Tatsache geschuldet, dass die für die Bewertung herangezogene Metaanalyse eine Reihe älterer Untersuchungen beinhaltet, bei denen nicht nur Patienten mit ACS, sondern auch Patienten mit stabiler KHK und sogar solche ohne koronarangiografischen Nachweis einer KHK berücksichtigt wurden.
Daher könne man auch angesichts einer neueren Metaanalyse, die einen deutlichen Nutzen für die längere duale Plättchenhemmung zeigte, die ESC-Bewertung nicht nachvollziehen.

Dr. med. Peter Stiefelhagen

Quelle: Expertenforum „ACS und mehr" am 03.10.15 in Berlin

\title{
Aktualisierte ESC-Leitlinie für NSTEMI
}

\section{Beim jüngsten Kongress der Europäischen Kardiologiegesellschaft (ESC) in London wurde eine aktualisierte Leitlinie für Nicht-ST-Hebungsinfarkt (NSTE- MI) vorgestellt. Neue Empfehlungen gibt es zum Zugangsweg, zur Troponin- Diagnostik und zum optimalen Timing und Dauer der dualen Plättchenhemmung.}

A rm ist besser als Leiste! So könnte man plakativ die Empfehlung für den optimalen Zugangsweg bei der Koronarintervention im Rahmen eines Nicht-ST-Hebungsinfarkts (NSTEMI) zusammenfassen. „Überraschend ist, dass durch den radialen Zugang nicht nur das lokale Blutungsrisiko, sondern auch das kardiovaskuläre Outcome und sogar die Gesamtmortalität gesenkt wird“, sagte Prof. Ulf Landmesser, Direktor der Kardiologischen Universitätsklinik der Charite Campus Benjamin Franklin in Berlin im Rahmen des Expertenforums „ACS uns mehr“ in Berlin.

Doch wie lässt sich das erklären? Beim femoralen Zugang treten häufiger lokale Blutungskomplikationen auf, sodass dann die duale Plättchenhemmung eventuell pausiert werden muss und der Patient nicht selten Bluttransfusionen benötigt. Dies alles wirkt prothrombogen, wodruch das Risiko für ein erneutes kardiovaskuläres Ereignis steigt.

1-Stunden-Protokoll schafft Klarheit Bisher gilt die Regel, bei Verdacht auf ein akutes Koronarsyndrom ohne ST-Streckenhebung (NST-ACS) sollte die Bestimmung des hs-Troponins zweimal in einem Abstand von drei Stunden erfolgen. In der neuen Leitlinie wird erstmals als Alternative ein kürzerer Algorithmus aufgeführt mit einer Kontrolle schon nach einer Stunde. Damit gelingt es bei einigen Patienten den NSTEMI früher auszuschließen oder nachzuweisen - aber nicht bei allen. Wenn bei der Kontrolle des hs-Troponins auch nach einer Stunde der Wert $<6 \mathrm{ng} / \mathrm{l}$ liegt, so ist ein NSTEMI mit fast $100 \%$ iger Sicherheit ausgeschlossen. Dazu gehören im klinischen Alltag ca. 40\% der Patienten mit Verdacht auf ein ACS. Liegt der Ausgangswert $>6 \mathrm{ng} / \mathrm{l}$ und steigt innerhalb der ersten Stunde auf mindestens $12 \mathrm{ng} / \mathrm{l}$ an, liegt die Wahrscheinlichkeit für einen NSTEMI bei 92,7\%. Diese Konstellation findet sich im Alltag bei $15 \%$ der Patienten. Diese Patienten müssen ins Katheterlabor. 50\% der Patienten bleiben jedoch in einem Graubereich und erfordern eine weitere Kontrolle nach drei Stunden.

\section{Preloading: Keine neue Empfehlung}

Die Frage, ob ein Preloading sinnvoll ist, wird seit vielen Jahren diskutiert. Für Clopidogrel gibt es lediglich Registerdaten, die darauf schließen, dass ein Preloading vorteilhaft sein könnte. Daher galt bisher die Empfehlung, Clopidogrel schon beim ersten Arztkontakt zu verabreichen. Prospektive Daten liegen aber nicht vor.

Für Ticagrelor gibt es keinerlei Daten beim NSTEMI. Im Falle von Prasugrel führt das Preloading beim NSTEMI nicht zu einem verbesserten Outcome. Die Blutungsereignisse stiegen aber deutlich an, sodass diese Substanz erst nach Kenntnis des Koronarbefundes, also direkt vor der Intervention, gegeben werden sollte.

\section{Dauer der Plättchenhemmung}

Unverändert bleibt die allgemeinen Empfehlung zur dualen Plättchenhemmung über zwölf Monate. Doch auch eine individualisierte Handhabung ist möglich. So kann die Dauer bei erhöhtem Blutungsrisiko, vor allem bei einer Tripletherapie auf drei bis sechs Monate verkürzt werden und bei hohem Risiko für ein erneutes ischämisches Ereignis kann die duale Plättchenhemmung bis $\mathrm{zu} 30$ Monate fortgeführt werden.

\section{Wann ins Katheterlabor?}

Was die Dringlichkeit der Koronarintervention beim NSTEMI betrifft, gibt es keine neuen Empfehlungen. Bei Patienten mit sehr hohen Risiken, also bei hämodynamischer Instabilität, anhaltenden Beschwerden, kardiogenem Schock, Herzinsuffizienz und malignen Arrhythmien sollte man den NSTEMI wie ein STEMI behanden, sprich die Koronarintervention sollte binnen zwei Stunden erfolgen.

Ein hohes Risiko liegt dann vor, wenn ein Myokardinfarkt bereits vorgekommen war, dynamische Endteilveränderungen vorliegen oder der GRACEScore $>140$ liegt. Solche Patienten sollten innerhalb von 24 Stunden eine Koronarintervention erhalten. Bei den übrigen Patienten mit einem intermediären $\mathrm{Ri}$ siko, wozu insbesondere Diabetiker gehören, sollte ein Zeitfenster von 72 Stunden eingehalten werden. 